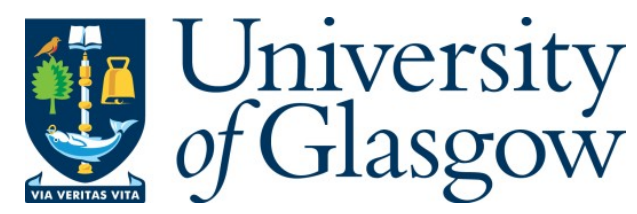

Schwarz, C. et al. (2012) The barrel DIRC of PANDA. Journal of Instrumentation, 7(2), C02008.

Copyright (C) 2012 IOP Publishing Ltd and SISSA

Version: Published

http://eprints.gla.ac.uk/85255

Deposited on: 02 July 2015

Enlighten - Research publications by members of the University of Glasgow http://eprints.gla.ac.uk 
The Barrel DIRC of PANDA

This content has been downloaded from IOPscience. Please scroll down to see the full text. 2012 JINST 7 C02008

(http://iopscience.iop.org/1748-0221/7/02/C02008)

View the table of contents for this issue, or go to the journal homepage for more

Download details:

IP Address: 130.209.115.202

This content was downloaded on 02/07/2015 at $11: 39$

Please note that terms and conditions apply. 
WORKSHOP ON FAST CHERENKOV DETECTORS - PHOTON DETECTION, DIRC DESIGN AND DAQ

April 4-6, 2011, Giessen, Germany

\section{The Barrel DIRC of PANDA}

\section{Schwarz $,{ }^{a}, 1$ A. Britting, ${ }^{b}$ P. Bühler, ${ }^{c}$ E. Cowie,${ }^{d}$ V.Kh. Dodokhov, ${ }^{e}$ M. Düren, ${ }^{f}$} W. Eyrich, ${ }^{b}$ K. Föhl, ${ }^{f}$ L. Gruber, ${ }^{c}$ A. Hayrapetyan, ${ }^{f}$ M. Hoek, ${ }^{d}$ R. Hohler, ${ }^{a}$ G. Kalicy, ${ }^{a}$ T. Keri, ${ }^{d}$ P. Koch, ${ }^{f}$ B. Kröck, ${ }^{f}$ A. Lehmann, ${ }^{b}$ D. Lehmann, ${ }^{a}$ B. Lewandowski, ${ }^{a}$ J. Marton, ${ }^{c}$ O. Merle, ${ }^{f}$ R. Montgomery, ${ }^{d}$ M. Patsyuk, ${ }^{a}$ K. Peters, ${ }^{a}$ G. Rosner, ${ }^{d}$ G. Schepers, ${ }^{a}$ L. Schmitt, ${ }^{a}$ J. Schwiening, ${ }^{a}$ B. Seitz, ${ }^{d}$ C. Sfienti, ${ }^{g}$ K. Suzuki, ${ }^{c}$ F. Uhlig, ${ }^{b}$ A.S. Vodopianov ${ }^{e}$ and E. Widmann ${ }^{c}$

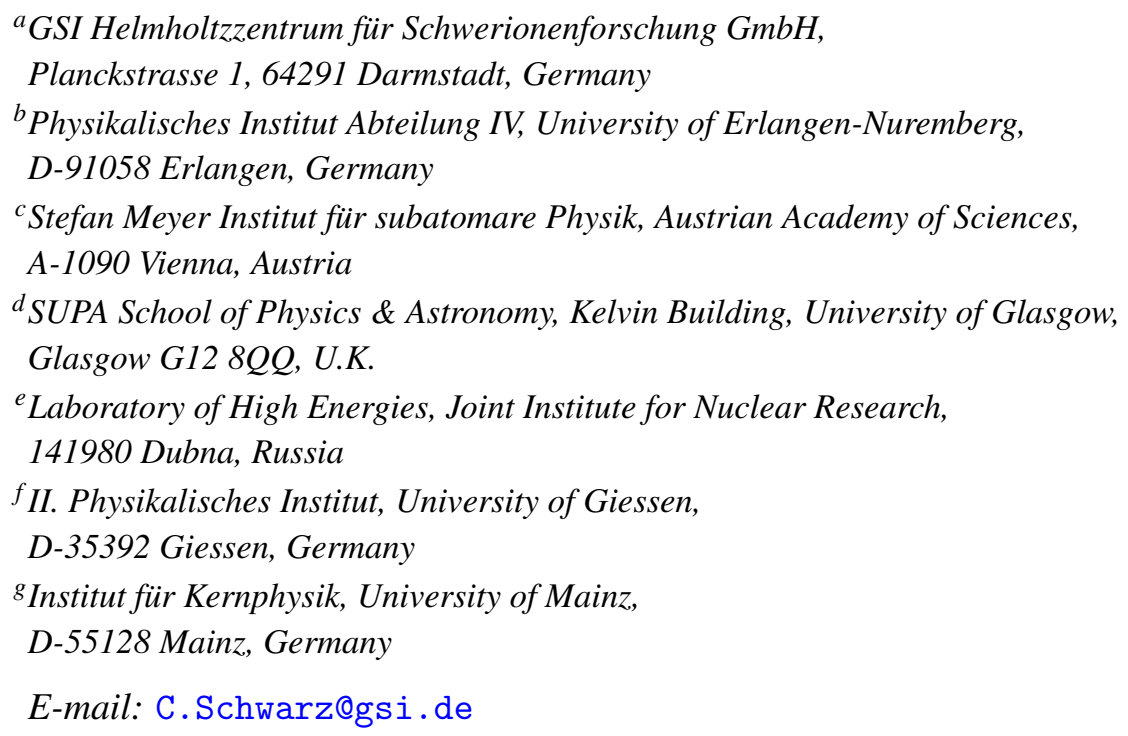

ABSTRACT: Cooled antiproton beams of unprecedented intensities in the momentum range of 1.5$15 \mathrm{GeV} / \mathrm{c}$ will be used for the PANDA experiment at FAIR to perform high precision experiments in the charmed quark sector. The PANDA detector will investigate antiproton annihilations with beams in the momentum range of $1.5 \mathrm{GeV} / \mathrm{c}$ to $15 \mathrm{GeV} / \mathrm{c}$ on a fixed target. An almost $4 \pi$ acceptance double spectrometer is divided in a forward spectrometer and a target spectrometer. The charged particle identification in the latter is performed by ring imaging Cherenkov counters employing the DIRC principle.

KEYWORDS: Particle identification methods; Cherenkov detectors

\footnotetext{
${ }^{1}$ Corresponding author.
} 


\section{Contents}

1 Introduction $\quad 1$

2 Barrel DIRC $\quad 2$

3 Performance $\quad 3$

4 Outlook 4

\section{Introduction}

The international Facility for Antiproton and Ion Research (FAIR) [1, 2] at GSI currently gets realized. A $30 \mathrm{GeV}$ proton synchrotron will provide secondary beams with unsurpassed quality to many experiments. Antiprotons abundantly produced at a rate of $2 \times 10^{7} \mathrm{~s}^{-1}$ will be accumulated and transferred into the High Energy Storage Ring (HESR). The design parameters cover the momentum range of 1.5 to $15 \mathrm{GeV} / \mathrm{c}$ with momentum resolution as good as $2 \times 10^{-5}$ when cooled either stochastically or by magnetized electron cooling. At the interaction point of the PANDA detector (antiProton ANnihilations at DArmstadt), shown in figure 1, this antiproton beam crosses a dense internal target such that the average luminosity reaches $2 \times 10^{32} \mathrm{~cm}^{-2} \mathrm{~s}^{-1}$ [3]. The interaction rate is $20 \mathrm{MHz}$ on average, but can reach up to $50 \mathrm{MHz}$ due to the filling scheme of the storage ring and fluctuations introduced by the target system. Two target systems are foreseen. A hydrogen cluster target with a steady target density in time and a pellet target with the capability to determine the primary interaction vertex.

The physics program of $\overline{\mathrm{P} A N D A}$ covers a wide range of subjects: High precision spectroscopy of resonances in the energy region of charmonium and above; the exact knowledge about masses, widths, and branching ratios provides information about the mechanisms of quark confinement; inmedium effects of open and hidden charm and their relationship to the chiral symmetry breaking addressing especially the origin of hadron mass; glueballs, predicted by QCD, need a firm experimental establishment in the current picture of strong interactions; hybrid meson states, also known as excited glue, in the mass range between 3 and $5 \mathrm{GeV} / \mathrm{c}^{2}$ are predicted to be rather narrow without mixing into neighboring resonances; hypernuclei, when abundantly produced, and examined with the next generation of $\gamma$-detectors, will revive the physics with single and double hypernuclei. This will improve the currently modest knowledge on their structure and yield information on the interaction of hyperons with nucleons as well as hyperon-hyperon interaction.

With the novel design of the detector as well as of the readout PANDA faces the challenges of the necessary momentum resolution (1\%), high dynamic range for $\gamma$-detection (few $\mathrm{MeV}$ to $10 \mathrm{GeV}$ ) and high-range charged-particle identification capability (several hundred $\mathrm{MeV}$ to some $\mathrm{GeV}$ for electrons, muons, pions, kaons, and protons). For the operation at the high interaction rate 


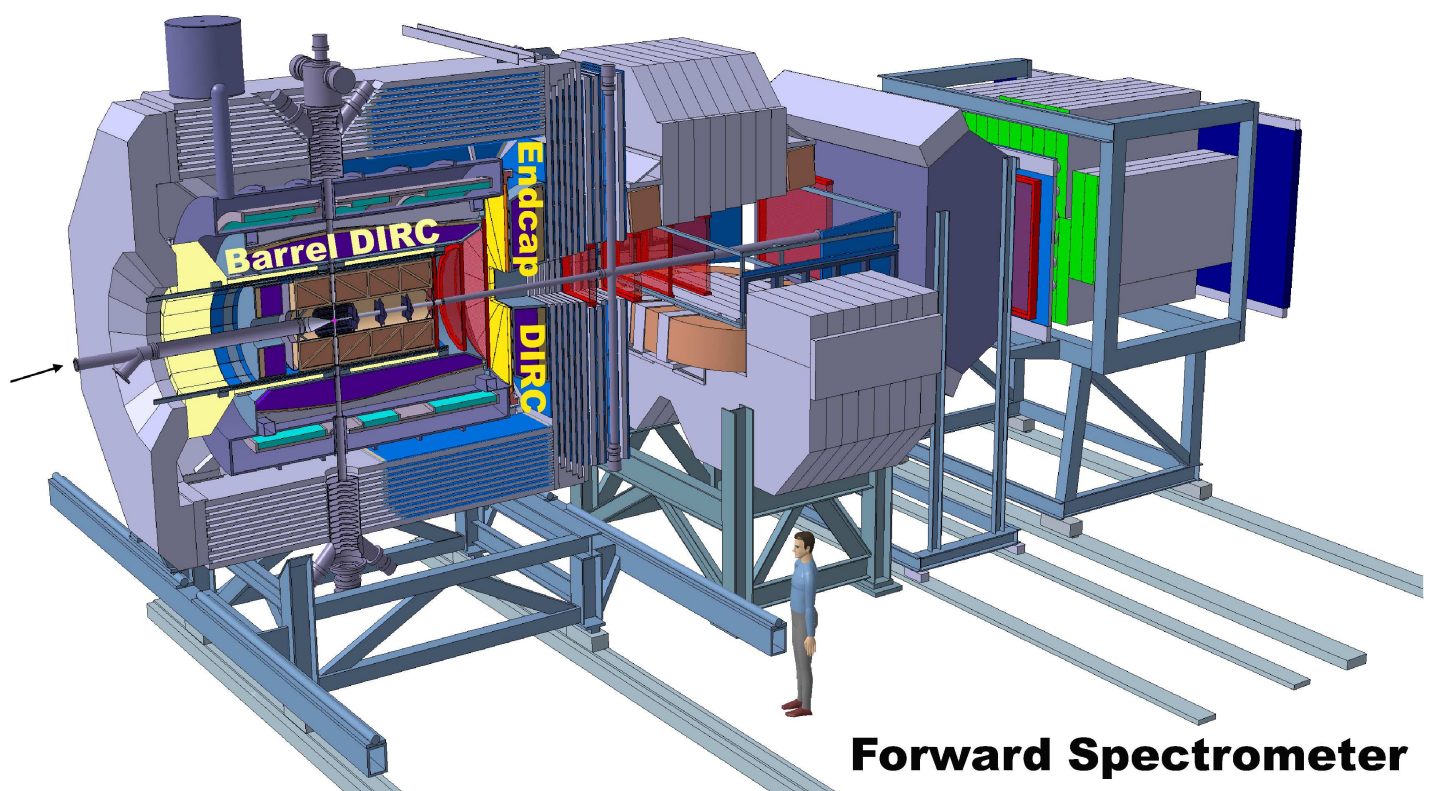

Target Spectrometer

Figure 1. The $\overline{\mathrm{P}} \mathrm{ANDA}$ detector comprises a target and a forward magnetic spectrometer. The figure shows the subdetectors within the solenoid and subdetectors inside and behind the dipole magnet of the forward spectrometer. The DIRC detectors in the target spectrometer are drawn in light colors. The beam comes from the left as indicated by the arrow.

of $2 \times 10^{7} \mathrm{~s}^{-1}$ a self-triggering readout scheme for different physical topologies is discussed where the frontend continuously processes the detector data and autonomously finds valid hits.

The detector, shown in figure 1 , is divided into a forward spectrometer with a dipole magnet and a target spectrometer with a solenoid magnet [5]. The Cherenkov counters within the target spectrometer take care of the charged particle identification. They are positioned between tracking detectors and an electromagnetic calorimeter consisting of $\mathrm{PbWO}_{4}$. In order to keep the latter small in size the Cherenkov detectors cannot occupy too much space [6,9]. Therefore, the DIRC principle was chosen for which the BaBar collaboration [7] has demonstrated an excellent performance. The target spectrometer houses a Barrel DIRC $[8,9]$ covering polar angles from $140^{\circ}$ down to $22^{\circ}$ and an Endcap DIRC, called the 3D Disc-DIRC, covering the angles from $22^{\circ}$ down to $10^{\circ}$ and $5^{\circ}$ in the horizontal and vertical directions, respectively (figure 2).

\section{Barrel DIRC}

When a charged particle traverses a radiator of refractive index $n$ with a velocity $\beta=v / c>1 / n$ it emits Cherenkov photons on a cone with a half opening angle $\cos \left(\theta_{C}\right)=1 /(\beta n)$. For particle tracks of $\beta \approx 1$ in radiators of refractive index $n>\sqrt{2}$ some photons are always totally internally reflected. The magnitude of the Cherenkov angle is conserved during the internal reflections provided adjacent and opposite optical surfaces are perpendicular and parallel to each other, respectively. The 


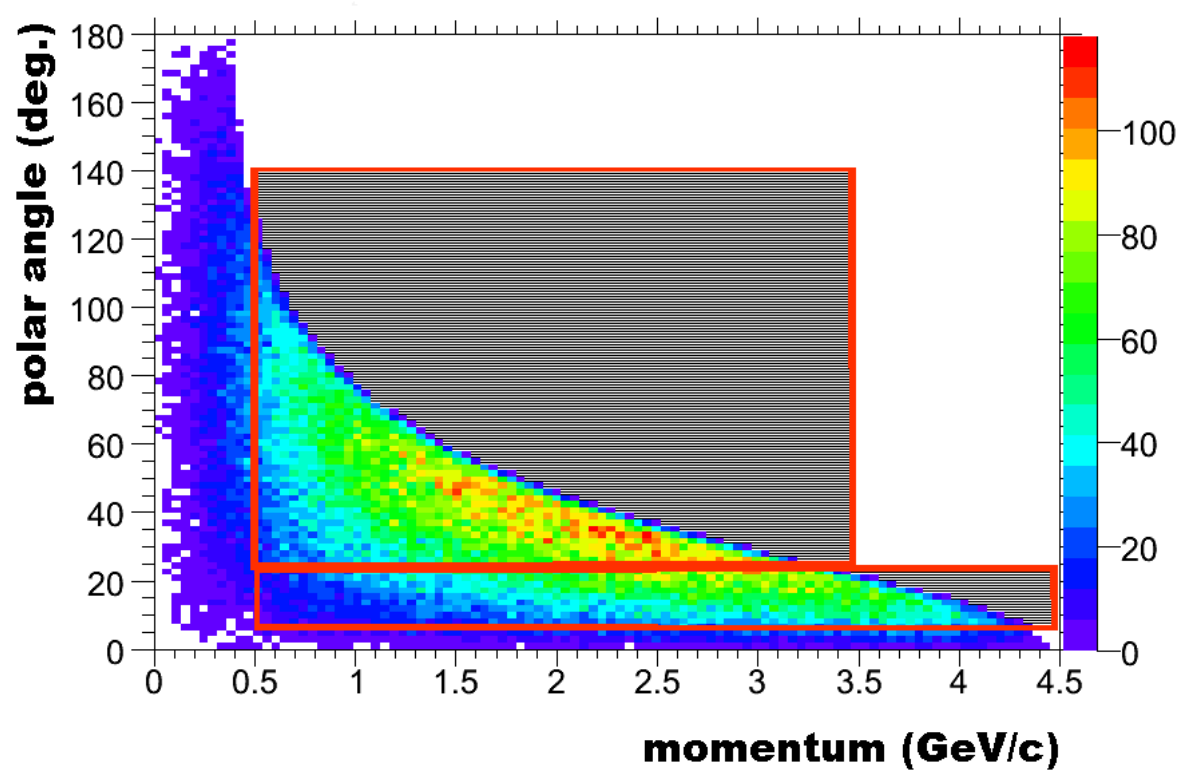

Figure 2. Polar angle distribution of kaons from the radiative decay $J / \psi \rightarrow \gamma K^{+} K^{-}$[4]. The rectangular boxes denote the acceptance of the Barrel DIRC and Endcap DIRC. The lower momentum threshold is the threshold for Cherenkov light generation.

efficiency for the DIRC to identify a kaon has been investigated with an downscaled setup of the $\mathrm{BaBar}$ [7] version for kaons in the mass region $450-550 \mathrm{MeV} / \mathrm{c}^{2}$. The efficiency to detect kaons is close to $100 \%$ with a pion misidentification at the level of $1-2 \%$. Operating the detectors in the veto mode, all low momentum tracks with momenta below the kaon threshold are considered to be kaons except those with Cherenkov signals. The purity of the kaon sample in this mode, however, depends on the detailed composition of the background. Currently the simulation of an improved design as shown in figure 1 is developed. In this design, a smaller photon expansion volume within the yoke of the solenoid magnet is used. Focussing lenses at the end of the radiator bars provide a sufficient sharp image on the photon detector plane. A the same time a fast reconstruction method is developed [10] to operate the DIRC within the online event selection. The simulation of a single charged particle within the PandaRoot framework [11] is shown in figure 3. The produced photons are internally reflected and guided towards the expansion box. Photons propagating towards the other end are reflected off a mirror. Depending on their direction, the photons reach different positions on the backside of the expansion box where they are recorded by photon detectors. From the hit position the initial photon direction can be calculated if the hit radiator is know (tracking detectors).

\section{Performance}

The current design is based on $2500 \times 17 \times 35 \mathrm{~mm}^{3}$ radiator bars made of synthetical fused silica. Five bars form one bar box and 16 bar boxes make up a barrel of $50 \mathrm{~cm}$ radius around the beam line. Two gaps, one on the top and one at the bottom of the barrel are foreseen for the vertical beam pipe, whereas all bar boxes are identical. The expansion box has a depth of $300 \mathrm{~mm}$ and the pixel size of 


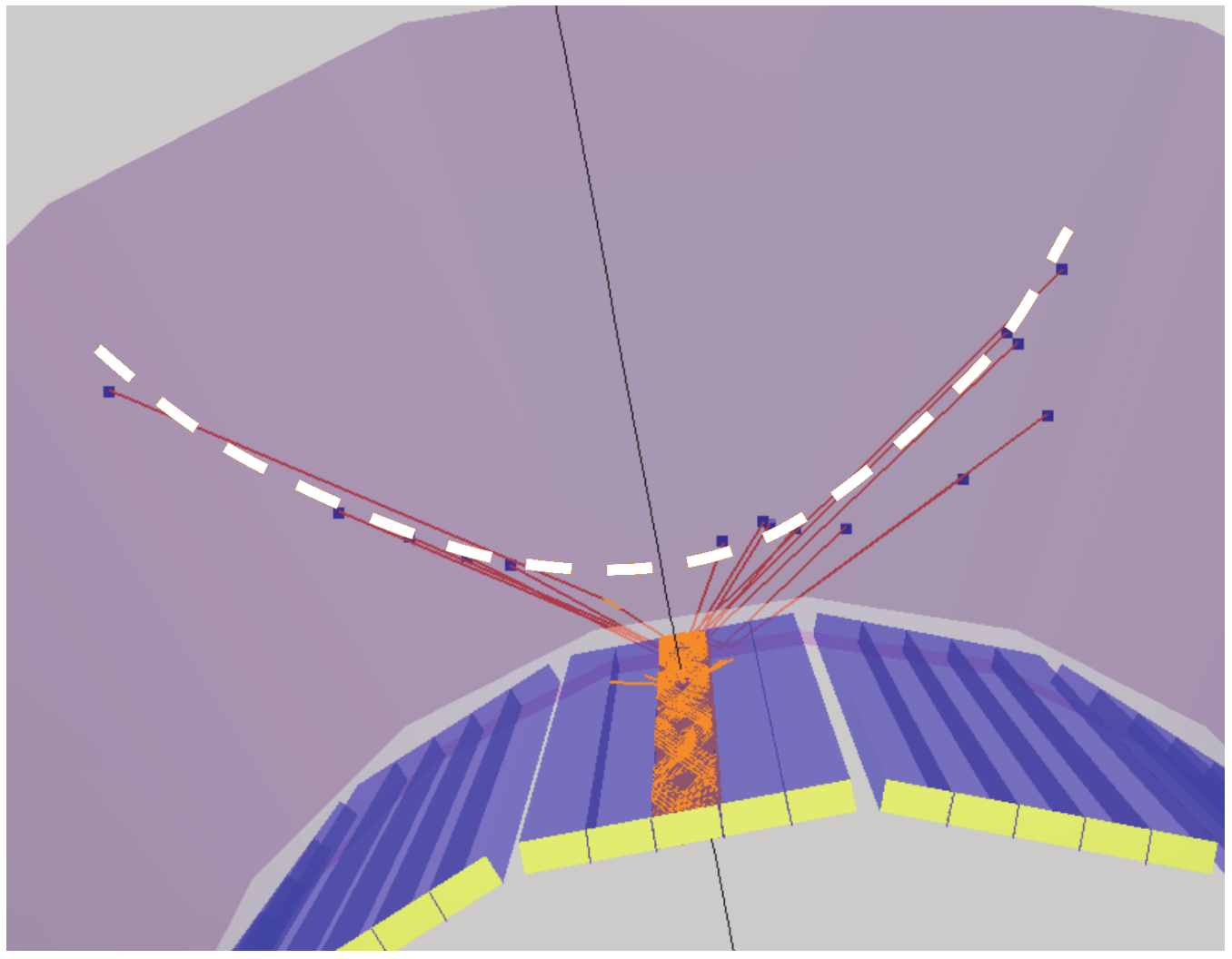

Figure 3. Upstream view of the $\bar{P} A N D A$ Barrel DIRC. The internallly reflecting photons propagate along the radiator until they hit the backside of the expansion box. Here, they are recorded by pixelized photon detectors.

the photon detectors is $6.5 \times 6.5 \mathrm{~mm}^{2}$ [13]. The expansion box is filled with Marcol82 oil [12] to reduce image distortions. Between radiator and expansion volume a focusing lens ensures a sharp image of the Cherenkov photons. The pixel size adds $6.3 \mathrm{mrad}$ to the single photon Cherenkov angle resolution. The chromatic angular resolution contributes for $5 \mathrm{mrad}$. These values dominate the combined single photon resolution that amounts to $8-9 \mathrm{mrad}$. For 20 photons and a track angular resolution of $1 \mathrm{mrad}$ an overall Cherenkov resolution of $2-2.5 \mathrm{mrad}$ is achieved. This allows the separation of pions and kaons with more than 3 standard deviations for the full momentum range up to $3.5 \mathrm{GeV} / \mathrm{c}$. Further details about the setup and geometry options can be found in ref. [14].

\section{Outlook}

The number of critical parameters of the current design are not yet optimized and several design options need to be investigated. The influence of these options on the performance of the DIRC, estimated in terms of single photon Cherenkov angle resolution and photon yield per particle, needs to be studies within the PANDARoot simulation framework. One of the design options is the cross section of the radiator which could be in the extreme case a plate. Another option is a different focussing system with a focussing mirror at the downstream end of the radiator. Most photons will 
hit this mirror since the angular distribution of the charged particles in a fixed target experiment is forward peaked.

\section{Acknowledgments}

This work is supported by EU6 grant, contract number 515873, DIRACsecondary-Beams, and EU FP7 grant, contract number 227431, HadronPhysics2.

\section{References}

[1] W.F. Henning, FAIR and its experimental program, J. Phys. G 34 (2007) S551.

[2] P. Spiller and G. Franchetti, The FAIR accelerator project at GSI, Nucl. Instrum. Meth. A 561 (2006) 305.

[3] PANDA collaboration, M. Kotulla et al., Strong interaction studies with antiprotons, letter of intent for PANDA (Antiproton Annihilations at Darmstadt), http://inspirehep.net/record/671592.

[4] M.S. Chanowitz and S.R. Sharpe, Glueballs and Meiktons which decay to multi-kaon final states, Phys. Lett. B 132 (1983) 413.

[5] PANDA collaboration, M.F.M. Lutz et al., Physics performance report for PANDA: strong interaction studies with antiprotons, arXiv:0903.3905.

[6] K. Föhl et al., The DIRC detectors of the PANDA experiment at FAIR, Nucl. Instrum. Meth. A 595 (2008) 88.

[7] BABAR-DIRC collaboration, I. Adam et al., The DIRC particle identification system for the BaBar experiment, Nucl. Instrum. Meth. A 538 (2005) 281.

[8] C. Schwarz et al., The Barrel DIRC of the PANDA experiment, Nucl. Instrum. Meth. A 595 (2008) 112.

[9] C. Schwarz et al., Particle identification for the PANDA detector, Nucl. Instrum. Meth. A 639 (2011) 169.

[10] M. Patsyuk et al., Simulation and reconstruction of the PANDA Barrel DIRC detector, 2011 JINST 6 C11015.

[11] S. Spataro et al., Simulation and event reconstruction inside the PandaRoot framework, J. Phys. Conf. Ser. 119 (2008) 032035.

[12] Exxon Mobil Corporation webpage, http://www.exxonmobil.com/.

[13] Burle-Planacon MCP-PMT, http://www.photonis.com/, http://www.burle.com/.

[14] PANDA CHERENKOV collaboration, J. Schwiening, The Barrel DIRC detector for the PANDA experiment at FAIR, Nucl. Instrum. Meth. A 639 (2011) 315. 